\title{
Alpha-band transcranial alternating current stimulation modulates precision, but not gain during whole-body spatial updating
}

\author{
T.P. Gutteling ${ }^{1}$, D.J.L.G. Schutter, W.P. Medendorp* \\ Radboud University, Donders Institute for Brain, Cognition and Behaviour, Montessorilaan 3, 6525 HR Nijmegen, The Netherlands
}

\section{A R T I C L E I N F O}

\section{Keywords:}

Spatial updating

Alpha

tACS

Remapping

Whole-body motion

Precision

\begin{abstract}
A B S T R A C T
Spatial updating is essential to maintain an accurate representation of our visual environment when we move. A neural mechanism that contributes to this ability is called remapping: the transfer of visual information from neural populations that code a location before the motion to those that encode it after the motion. While there is ample evidence for neural remapping in conjunction with eye movements, only recent findings suggest a role of this mechanism for whole-body motion updating, based on the observation that alpha band (10 Hz) activity is selectively reorganized during remapping. This study tested whether alpha oscillations directly contribute to whole-body motion updating using transcranial alternating current stimulation (tACS). In a double blind sham controlled design, healthy volunteers received $10 \mathrm{~Hz}$ tACS at an intensity of $1 \mathrm{~mA}$ over either the left or right posterior hemisphere during a whole-body motion updating task. Updating performance was assessed psychometrically and indices of gain and precision were obtained. No tACS-related effects on updating gain were found, irrespective of whether the remapping was across or within the hemispheres. In contrast, updating precision was enhanced when a target representation had to be internally remapped to the stimulated hemisphere, but not in other remapping conditions. Our observations suggest that alpha band oscillations do not directly affect the transfer of target representations during remapping, but increase the fidelity of the updated representation by attenuating interference of afferent information.
\end{abstract}

\section{Introduction}

When navigating through the environment, we continuously keep track of objects in our immediate surroundings, internally updating their locations in conjunction with our moving body i.e. 'spatial updating' (for reviews see Klier and Angelaki, 2008; Medendorp, 2011). The neural correlates of this behavior have been studied extensively using saccadic updating paradigms. Based on monkey single-unit studies, one mechanism that has been identified is called remapping: the transfer of the representation of a stimulus location from neurons activated by the stimulus before the saccade to those that represent its location after the saccade (Colby et al., 1995; Sommer and Wurtz, 2008). Evidence for this mechanism has also been found in the human brain, based on brain oscillations (Van Der Werf et al., 2013), BOLD signals (Medendorp et al., 2003; Merriam et al., 2003) and TMS findings (Morris et al., 2007).

In contrast to saccades, the neural underpinnings of spatial updating across head and body motion have been studied much less. It is only recently that studies suggest that remapping can also account for updating across whole-body motion (Gutteling et al., 2015; Gutteling and Medendorp, 2016). Evidence is based on neural oscillations in the alpha band $(8-12 \mathrm{~Hz})$, whose power increases and decreases have been associated with the selective inhibition and engagement of neuronal populations, respectively (Jensen and Mazaheri, 2010).

In Gutteling et al. (2015), we showed that alpha band power across occipital-parietal areas switches hemispheres when a target location reverses sides relative to the body midline (which was aligned with gaze) during the motion. More specifically, an increase of power was observed in the hemisphere to which the target representation was remapped. These findings were interpreted as a protection mechanism in which alpha oscillations impede other inputs that could distort the quality of target representation (Gutteling and Medendorp, 2016). Furthermore, a correlation was observed between the change in power and the spatial extent of remapping, as if alpha oscillations facilitated transfer of the target representation from the one neuronal population to the other during remapping. However, it remains unclear whether alpha oscillations directly gate remapping, reduce interference effects, or only reflect a proxy outcome of the remapping process.

\footnotetext{
* Corresponding author.

E-mail addresses: t.p.gutteling@bham.ac.uk (T.P. Gutteling), d.schutter@donders.ru.nl (D.J.L.G. Schutter), p.medendorp@donders.ru.nl (W.P. Medendorp).

${ }^{1}$ Present address: University of Birmingham, School of Psychology, Centre for Human Brain Health, Birmingham B15 2TT, United Kingdom.
} 
A
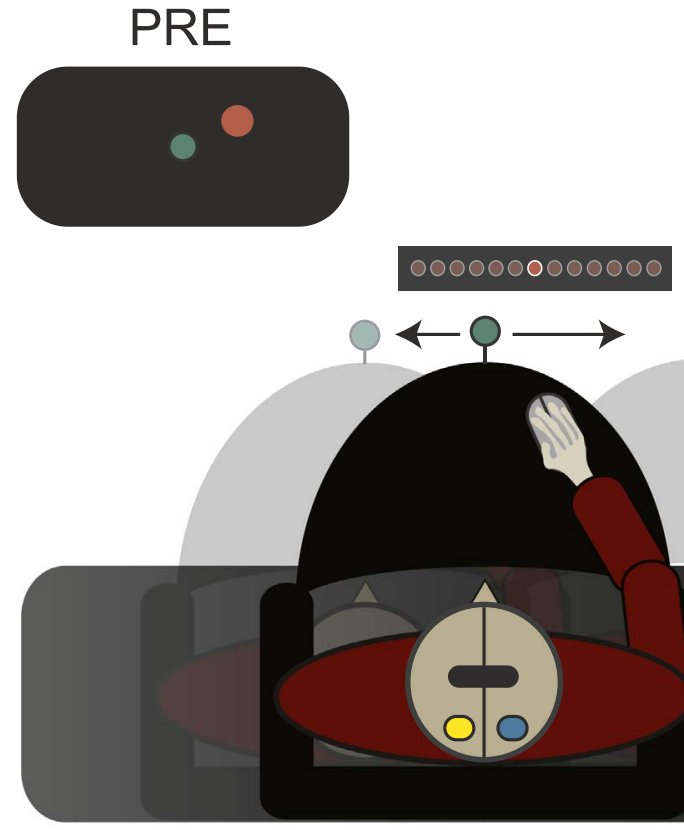

B
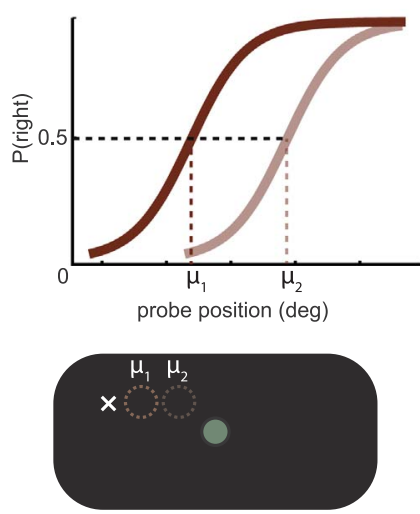

$\mathrm{D}$

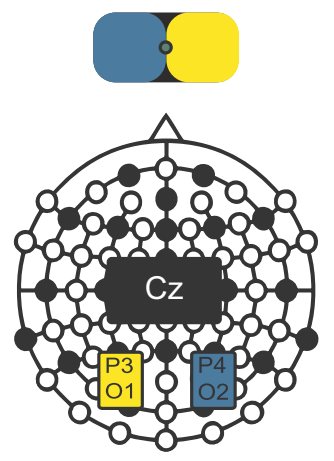

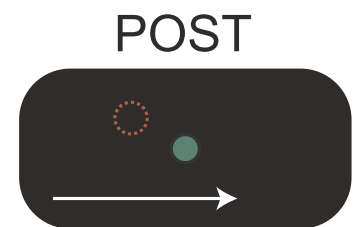

Across hemifields

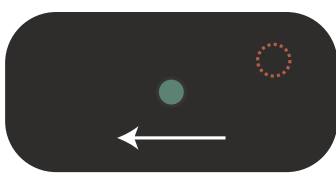

Within hemifield
C
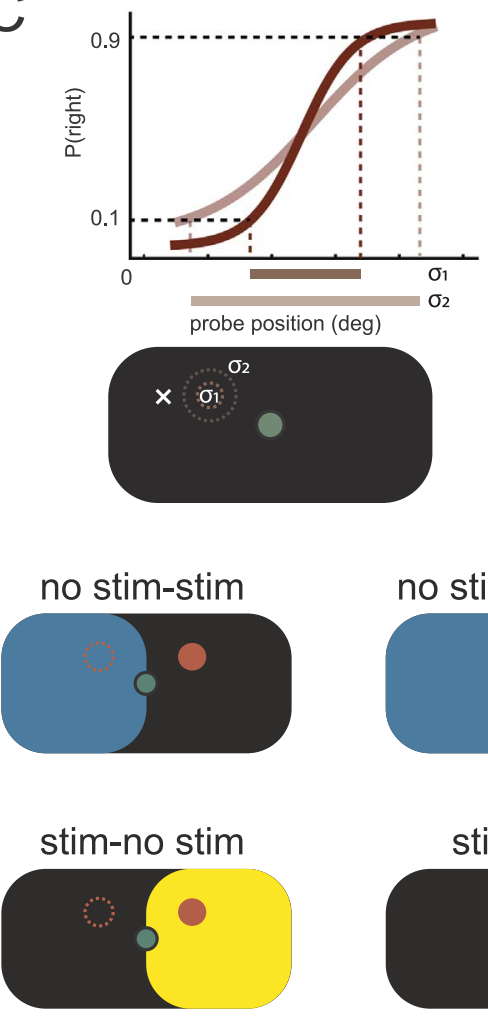

no stim-no stim
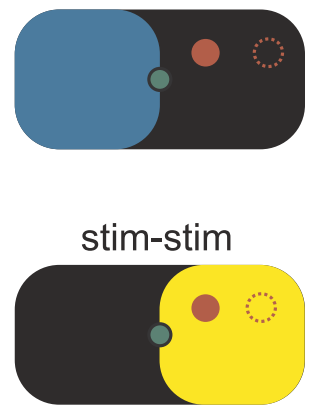

Fig. 1. Overview of the paradigm. A) Schematic top view of the experimental setup. Subjects were seated in the translation sled, in complete darkness, while a target (red circle) was presented using an LED array in front of the participant. Fixation was attached to the sled (green circle) and remained straight ahead of the subject. After target presentation, the sled moved either toward or away from the remembered target, resulting in an updated target either being remapped across hemifields, or within. Insets show the subjects' view before body motion (pre, left) and after (post, right). Dotted circles denote remembered (non-visible) targets. B) Derivation of the gain parameter and $\mathrm{C}$ ) the variance from the psychometric curve. The gain is derived from the mean (50\% point) of the curve and corrected for movement distance (see Section 2). In this paradigm, gain affects the remembered location of the spatial target. The variance is derived from the slope of the curve and affects the precision of the target location. D) Montage arrangement of the electrical stimulation. A small electrode was placed over either the left or right parieto-occipital areas, corresponding to EEG 10-20 electrode location $\mathrm{P} 3 / \mathrm{O} 1$ and $\mathrm{P} 4 / \mathrm{O} 2$ respectively. A larger electrode was placed over $\mathrm{Cz}$. E) Overview of the experimental conditions from the subjects' view. Red filled targets represent visual (initial) targets, dotted circles represent remembered, remapped targets after the motion. Targets had to be remapped either to the other hemifield, or within the same hemifield. Conditions are named after the position of the initially presented target and remembered target relative to stimulation, e.g. in the no stim-stim condition the initial target appears in the hemifield contralateral to the nonstimulated hemisphere and the remembered target contralateral to the stimulated hemisphere. Colored hemifields denote the stimulated hemifield according to the montage in D. (For interpretation of the references to color in this figure legend, the reader is referred to the web version of this article.)
Given that spatial updating is reflected in a specific spatiotemporal pattern of alpha band activity, we applied transcranial alternating current (tACS) at the alpha frequency $(10 \mathrm{~Hz})$ unilaterally over either left- or right occipital-parietal areas in a task that requires spatial updating during whole-body motion. We assessed the effect of stimulation psychometrically and derived two behavioral measures (see Fig. 1B): the updating gain, reflecting the amount of internal transfer of the remembered spatial location (i.e., the estimated spatial shift of a location), and updating precision, a measure for the quality of the remembered spatial location (i.e., the non-systematic variance in location). The gain and precision updating may reflect different mechanisms and be independently affected.
Previous studies have shown that a low current applied to the scalp, alternating at alpha frequency can entrain alpha band activity (Ruhnau et al., 2016; Zaehle et al., 2010), and cause behavioral effects in various cognitive domains (Brinkman et al., 2016; Cecere et al., 2015; Helfrich et al., 2014; Schutter and Wischnewski, 2016), although the exact mechanisms are in dispute (Herrmann et al., 2013; Kar and Krekelberg, 2014; Vossen et al., 2015).

Based on our recent observations (Gutteling and Medendorp, 2016), we hypothesize that alpha band is directly involved in the remapping, either as an interhemispheric transfer mechanisms and/ or an interference protection mechanism. Given this, alpha tACS will improve the updating gain for representations that are remapped across the 
hemispheres by facilitating transfer. If the alpha oscillations are actively involved in reducing interference, updating precision should be enhanced for targets that are remapped from the non-stimulated- to the stimulated hemisphere during alpha tACS, better protecting the representation fidelity.

\section{Material and methods}

\subsection{Participants}

Thirty healthy volunteers (16 women, mean age 23.6 yrs, range 18-31) participated in the study. Inclusion criteria were right-handedness, non-smoking, and normal or corrected-to-normal vision. Exclusion criteria were disorders of the visual and motor system, metal in cranium, use of medication (i.e., anti-epileptics, neuroleptics, tricyclic antidepressants or benzodiazepines), first degree epilepsy or family history of epilepsy, history of closed-head injury, history of head surgery, history of neurological or psychiatric disorders, medication pump, brain infarction, heart disease, cardiac pacemaker, pregnancy, and electronic hearing devices. Subjects were asked not to consume alcohol $24 \mathrm{~h}$ - and no caffeine or chocolate $2 \mathrm{~h}$ prior to the experiment. Written informed consent was obtained and subjects received payment or course credit for participation. The study was performed in accordance with the standards set by the Declaration of Helsinki and approved by the medical ethical review board of Arnhem-Nijmegen.

\subsection{Setup}

All measurements took place in a completely darkened room. Subjects were seated in a custom-made linear sled capable of translating sideways along an $800 \mathrm{~mm}$ track. The sled, powered by a linear motor (TB15N, Technotion, Almelo, The Netherlands), was controlled by a Kollmorgen S700 (Danaher, Washington, DC) drive. The sled was controlled with an accuracy better than $50 \mu \mathrm{m}, 2 \mathrm{~mm} / \mathrm{s}$, and $150 \mathrm{~mm} /$ $s^{2}$. The subjects' interaural axis was aligned with the sled motion axis. Participants were restrained with a five-point seat belt. Head motion was restrained with a modified over-ear headphone fixed to the sled. Responses were given using a computer mouse (see paradigm) in the subjects' right hand. During the task, white noise was presented using in-ear earphones, masking the sound produced by sled motion.

For each subject, the experiment consisted of two separate sessions, at least one week apart. Both sessions started at the same time of day. In every session, the updating task (lasting about 20-30 min) was performed twice: once during a pre-test to establish a baseline, and once during a stimulation session. The pre-test was included to be able to compensate for week-to-week variation in performance within subjects. During the stimulation session, transcranial alternating current stimulation (tACS) with a frequency of $10 \mathrm{~Hz}$ (alpha band central frequency) was applied as either real or sham stimulation using a battery-driven current stimulator (Eldith DC Stimulator, Ilmenau). The current was ramped up to $1 \mathrm{~mA}$ (peak-to-peak) over $10 \mathrm{~s}$. In the sham condition, the current was ramped down again after $1 \mathrm{~min}$ while the current remained on in the stimulation condition. The experiment was performed doubleblind regarding the stimulation condition, that is, the experimenter did not know whether the stimulation was real or sham. The stimulator is operated by entering a digit code that results in sham or real stimulation. Two codes were generated for every subject and session. Neither subject nor experimenter could derive the real of sham stimulation from the code and every code was used only once. Double-blind coding was performed using the open-source web-based application RANDI2 (www.randi2.org), performed by one of the staff members who was not directly involved in data acquisition.

The stimulation covered either the left or right parieto-occipital areas because these regions have shown the largest alpha band modulation in previous updating experiments (Gutteling et al., 2015; Gutteling and Medendorp, 2016). Subjects were pseudorandomly assigned to either the left or right montage group, while counterbalancing gender between groups. One electrode $(4 \times 3 \mathrm{~cm})$ was placed at EEG electrode position P3-O1 (10-20 system) for a left montage or P4-O2 for a right montage. The second electrode $(9 \times$ $5 \mathrm{~cm}$ ) was placed over electrode $\mathrm{Cz}$ (vertex), see Fig. 1D. The vertex electrode was considerably larger to reduce the focality, and thus effectiveness, of stimulation in this area of no-interest. Skin contact was ensured using conductive gel (SuperVisc gel, Easycap GmbH). Impedance was typically kept below $10 \mathrm{k} \Omega$. Depending on the subject's performance, duration of tACS was typically $25 \mathrm{~min}(+/-5 \mathrm{~min})$. None of the participants of the actual experiment reported phosphenes during tACS (Schutter, 2015). Some participants noticed a slight tingling sensation at the onset of stimulation, which dissipated quickly. However, this occurred in the initial phase of both real and sham stimulation.

Binocular eye movements were recorded at $500 \mathrm{~Hz}$ using an EyeLink 1000 system (SR research, Kanata, Canada), with online head motion correction. The camera system was mounted on the sled and used infrared illumination outside the visible spectrum.

Visual stimuli were presented using red light-emitting diodes (LEDs). A single LED was mounted on the sled at $\sim 720 \mathrm{~mm}$ from the subjects' eyes and served as a body-fixed fixation point. A 450-mm-wide array of LEDs, consisting of 180 LEDs with a spatial separation of $2.5 \mathrm{~mm}$ (center to center), was used to present target and probe stimuli. The LEDs were approximately square $(2 \times 2 \mathrm{~mm})$, USB-powered with a luminance of $1 \mathrm{~cd} / \mathrm{m}^{2}$ or less. This array was oriented in parallel with the sled movement axis, and was mounted on a tripod on the floor where it was stationary at $900 \mathrm{~mm}$ from the subjects' eyes. The fixation point and LED array were displaced vertically by a few millimeters, such that they did not occlude each other and were approximately at eye level.

\subsection{Paradigm}

The task consisted of a spatial updating paradigm with world-fixed target locations and a body-fixed fixation point, similar to (Gutteling and Medendorp, 2016), see Fig. 1A. At the start of each trial, the fixation led that was mounted on the sled, straight ahead of the subjects, was turned on. This forced the subjects to fixate straight ahead throughout the entire trial, which was confirmed by eye tracking. After $0.5 \mathrm{~s}$, a target was presented either to the left or right of the central fixation point for $100 \mathrm{~ms}$ (thus in the left or right hemifield), which had to be remembered across body motion. The target was always presented $2.9^{\circ}(45 \mathrm{~mm})+/-0.3^{\circ}(5 \mathrm{~mm})$ jitter to the left or right using a LED array. After another $500 \mathrm{~ms}$, subjects were passively translated sideways for $1 \mathrm{~s}$ using a minimum jerk profile, while subjects (internally) kept track of the remembered target location. This motion profile minimizes the third derivative of position (the rate of acceleration), which yields very smooth motion. The motion could either be in the direction of the target, or away from it. When moving towards the target, the displacement was $180 \mathrm{~mm}$ (peak velocity $340 \mathrm{~mm} / \mathrm{s}$ ), and the target ended up $135 \mathrm{~mm}\left(8.5^{\circ}\right)$ on the other side of the fixation point, thus in the opposite hemifield. So, relative to the body midline and gaze direction, the remembered location moved across hemifields. When moving away from the target, the displacement was $90 \mathrm{~mm}$ (peak velocity $170 \mathrm{~mm} / \mathrm{s}$ ), also ending up $135 \mathrm{~mm}\left(8.5^{\circ}\right)$ away from fixation, on the same side. So, relative to the body midline and gaze direction, the remembered location shifted within the same hemifield. After the motion and a subsequent $500 \mathrm{~ms}$ post-motion period, a world-fixed probe LED was flashed. The subjects' task was to indicate whether the probe was to the left or right of the initial target using a mouse in the right hand. After clicking the mouse (left or right button), a new trial began. If subjects failed to see either the target or probe flash, the middle mouse button could be pressed to withhold a response. The mouse response was made without time pressure.

The probe location was varied systematically to assess the 
remembered target location psychometrically (see Analysis). A fixedinterval procedure was used, equally spaced over 9 locations, spanning 8 degrees of visual angle, with a maximum of 12 degrees depending on subject performance. Due to the general underestimation of self-motion in these updating tasks (Clemens et al., 2012; Gutteling et al., 2015) probe locations were shifted towards the fixation point (consistent with an underestimation of motion), but always contained the veridical location.

Subjects were instructed to remember the position of the initial target as if it is fixed in the world, update its memorized location over the course of the sled motion, and compare the internally updated location with the location of a probe ('is it left or right of the first one?'). There was a small (0.5-1 min) break every 54 trials, during which the lights were turned on. On average, subjects completed 5 of these small blocks, yielding $\sim 270$ trials, depending on the speed of response of subjects. In total, the subjects performed the task four times (pre-test and stimulation, for two separate sessions), totaling up to $\sim 1080$ trials per subject. In total, for every subject, there were sixteen conditions as the result of two stimulation conditions (sham/real), two test periods (pre-test/stimulation), two updating conditions (within/across hemifields) and two displacement directions (left/right). Because there were two possible stimulation montages (left or right hemisphere), the initial target could appear in either the stimulated or non-stimulated hemifield, see Fig. 1E. Updating conditions and laterality of the initial targets were randomized, with the restriction that the same motion direction could only be repeated once (e.g. motion to the left twice was always followed by a movement to the right). For clarity, we refer to the location of the initial and remapped stimulus relative to stimulated hemifield when naming conditions. We assumed that stimulation predominantly affects the contralateral hemifield. Thus, a subject in the left hemisphere stimulation group (stimulating the right hemifield), being presented with a target on the right, which is on the left after the motion (updating across) is in a 'stimulation to no-stimulation', or 'stimno stim' condition. Data were sorted and averaged according to laterality of the initial target regarding the stimulated hemifield (stim/no stim) and updating condition (across/within), yielding four conditions per stimulation type: 'stim-stim', 'no stim-no stim' (updating within hemifield), 'no stim-stim' and 'stim-no stim' (updating across hemifields).

\subsection{Analysis}

This study used psychophysics with a fixed-stimulus interval (see 'paradigm'). Responses outside the stimulus range are indicative of erroneous performance. When subjects' performance was so poor that their responses were beyond the stimulus range, they were excluded due to failure to perform the task. Eight participants were excluded from the analysis due to: not finishing the experiment (2), equipment failure (1), failure to perform the task (5). One subject did not tolerate the initial tingling sensation caused by the tACS, all others tolerated stimulation well and reported no noticeable sensation after the initial current ramping up. For the remaining subjects (12 women, mean age 24.1 yrs, range 18-31), analyses were performed using MATLAB (Mathworks, Natick, MA, USA). Subjects' responses were used to calculate the likelihood of a right response as a function of probe location by fitting a logistic function using a Bayesian inference approach for each task condition using psignifit3 (Fründ et al., 2011). The mean of this function $(\mu)$ represents the bias (positive value corresponds to a rightward bias), see Fig. 1B. The width of the curve ( $\sigma)$, determined over the interval in which the function rises from $0.1(\alpha)$ to $0.9(1-\alpha)$, serves as a measure of the participant's variability in the updating task and is inversely related to precision, see Fig. 1C. All statistics were performed using gain and variance measures. To measure updating accuracy, independent of the direction or size of the motion (D), the updating gain $(\gamma)$ was calculated. As the displacement size is identical to the target displacement (from the subjects' perspective), the estimated displacement $\hat{D}=D-\mu$, and $\gamma$ is calculated as $\hat{D} / D$. This way, the gain factor is 1 for ideal updating performance and 0 when no updates are made. This gain factor represents the accuracy of the update and can be seen as a measure for transfer between neural populations coding spatial locations during remapping. To test the actual stimulation effects in each condition, but also take into account the baseline performance, the accuracy and variance values were entered in a repeated measures ANOVA with factors 'stimulation type' (2, sham/ actual), 'time' (2, pre-test/stimulation), 'condition' (no stim-no stim/ stim-stim/no-stim-stim/stim-no stim) with an alpha level of significance at 0.05 . Post-hoc $t$-test were used for further testing.

\section{Results}

We assessed spatial updating performance during whole-body selfmotion, while $10 \mathrm{~Hz}$ tACS at $1 \mathrm{~mA}$ (peak-to-peak) was applied over either left or right parieto-occipital areas. Subjects had to remember a world-fixed target that was briefly flashed prior to a passive displacement of the body and report its remembered location after the motion using a $2 \mathrm{AFC}$ response (i.e., whether a probe was perceived left or right of the memorized target).

From the psychometric results we calculated estimates of the bias $(\mu)$ in each of the four conditions, which we converted into an updating gain ( $\gamma$, see Methods). We also derived the slope of the psychometric curves, which is a measure for the variance $(\sigma)$ of the response, which

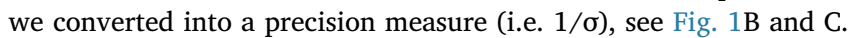

Fig. 2 shows the results of the updating gain, averaged across subjects (black), superimposed on the single subject results (in gray), during actual stimulation (upper panel) and sham stimulation (bottom panel). Both panels also demonstrate the pre-test gains, i.e. without stimulation at all. A repeated measures ANOVA with factors 'stimulation type' (2, sham/actual), 'time' (2, pre-test/stimulation), and 'condition' (4) revealed a significant main effect of 'time', $\left(\mathrm{F}_{(1,21)}=5.35, \mathrm{p}\right.$ $=0.03$ ), indicating that the mean gain in the pre-tests was higher than in the stimulation conditions (pre-tests: $\gamma=0.36, \mathrm{SD}=0.23$ vs stimulation: $\gamma=0.32$, SD $=0.22$ ). This session order effect occurred regardless of whether tACS was real or sham. No other significant main or interaction effects were observed $(0.13>\mathrm{p}>0.84)$. So, alpha tACS did not affect the updating gain.

Fig. 3 shows the effects of alpha tACS on updating precision, averaged across subjects (in black), as well as the single subject results (in gray), both for actual stimulation (upper panel) and for sham stimulation (bottom panel). An ANOVA showed a significant 'stimulation type' $\mathrm{x}$ 'time' $\mathrm{x}$ 'condition' interaction $\left(\mathrm{F}_{(3,19)}=3.45, \mathrm{p}=0.04\right)$, which is equivalent to a significant 'stimulation type' $\mathrm{x}$ 'condition' interaction based on the pre-test - stimulation difference scores (baseline correction). There were no other significant effects (all $\mathrm{p}>0.06$ ). The threeway interaction was further analyzed using post-hoc paired sample $t$ tests. As we were interested in the effect of actual stimulation, we tested whether the stimulation effect, i.e. the difference between pre-test and stimulation, differed between actual and sham stimulation. This resulted in 4 tests, one for each condition. A significant stimulation-related effect in the 'no stim-stim' condition was observed (stimulation effect, actual: 1.60 sham: $-0.60, t=3.21, p=0.017$, Bonferroni corrected). No other post-hoc tests were significant (all $\mathrm{p}>0.42$, uncorrected). These results indicate that the precision of updating is enhanced when the target has to be remapped from the non-stimulated to the stimulated hemisphere. This is illustrated in Fig. 4, where the difference between stimulation and pre-test is plotted as a function of stimulation type. As can be seen, the mean is clearly above unity in the no stim-stim condition.

To examine the extent to which the differences in the pre-test conditions could explain the effect of real versus sham tACS, we performed a number of additional exploratory analyses. We first performed a repeated measures ANOVA on only the pre-test data with factors 'stimulation type' and 'condition'. No significant main effects or 


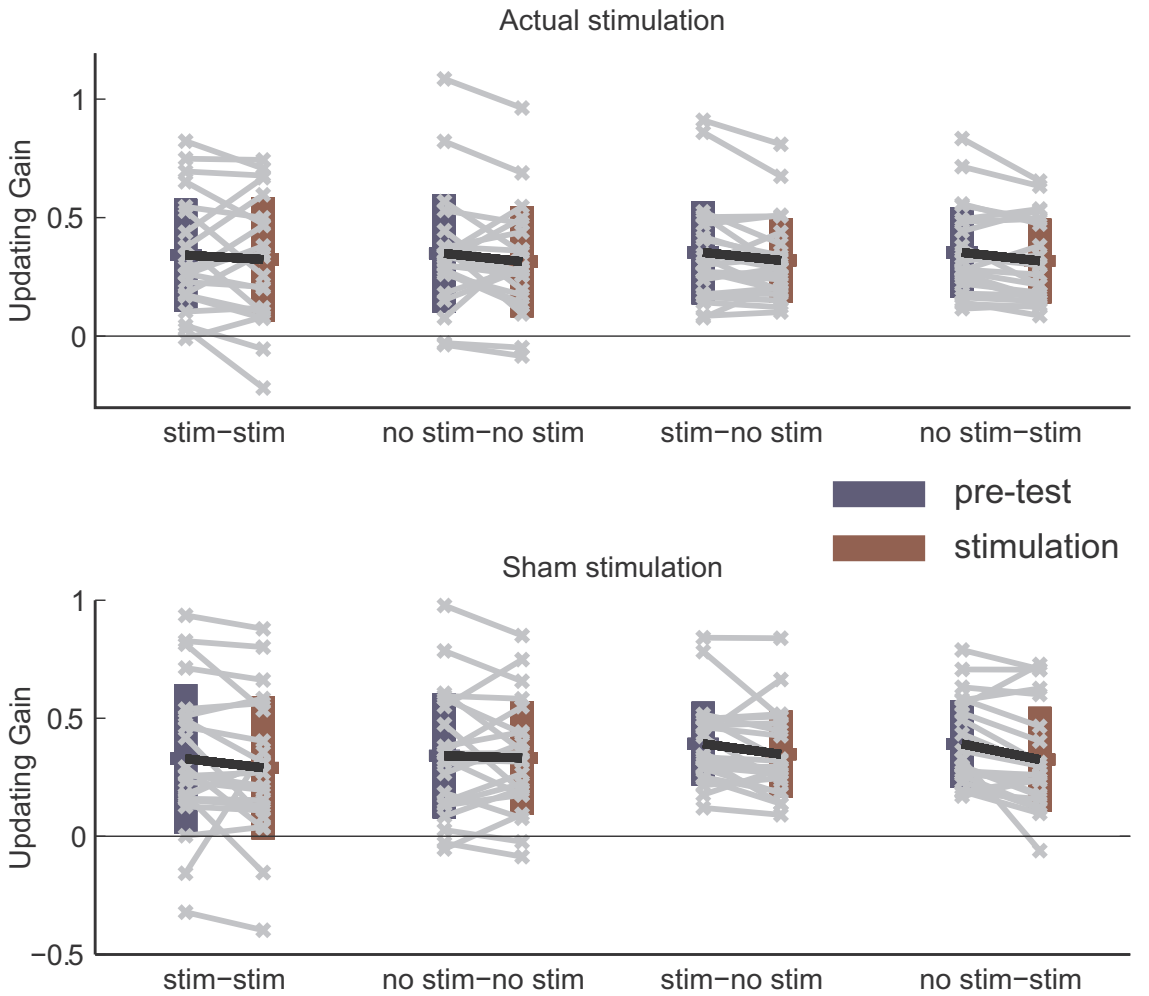

Fig. 2. Updating gain group results. Group averages for the gain factor with actual stimulation (A) or sham (control) stimulation (B) for all conditions. Vertical colored bars denote \pm 1 standard deviation from the mean. Gray lines are individual subject data; the black line denotes the mean. Updating gain was significantly reduced in the second session of the day (pre-test vs. stimulation). No other significant effects were found. interactions were found (all $\mathrm{p}>0.06$ ). We then split the data into a 'sham stimulation first' and an 'actual stimulation first' group. This revealed no significant pre-test differences between these groups for the actual stimulation data (independent samples $t$-test, equal variances not assumed; $0.09<\mathrm{p}<0.98$, uncorrected), or for the sham stimulation data $(0.12<\mathrm{p}<0.62$, uncorrected). There were also no significant pre-test differences between males and females $(0.10<\mathrm{p}<0.95$, uncorrected). Furthermore the effect is not driven by a single or a few subjects, but is quite systematic as precision is enhanced for 17 out of 22 subjects $(77 \%)$ with actual stimulation. Finally, we pooled pre-test performance of the across hemifield conditions, which should yield similar performance, to reduce variance in the pre-tests. Using this pretest baseline, our main post-hoc test remained significant $(\mathrm{t}=3.03$, $\mathrm{p}$ $=0.024$, same correction).

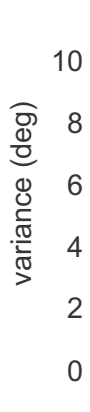

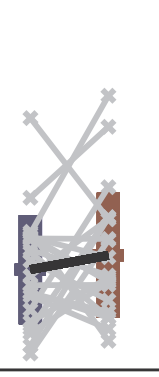

stim-stim
Actual stimulation

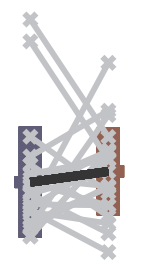

no stim-no stim

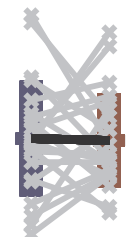

stim-no stim

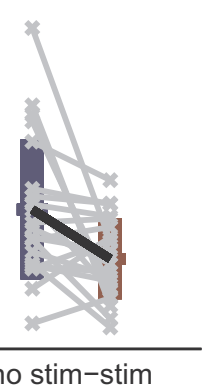

pre-test

stimulation

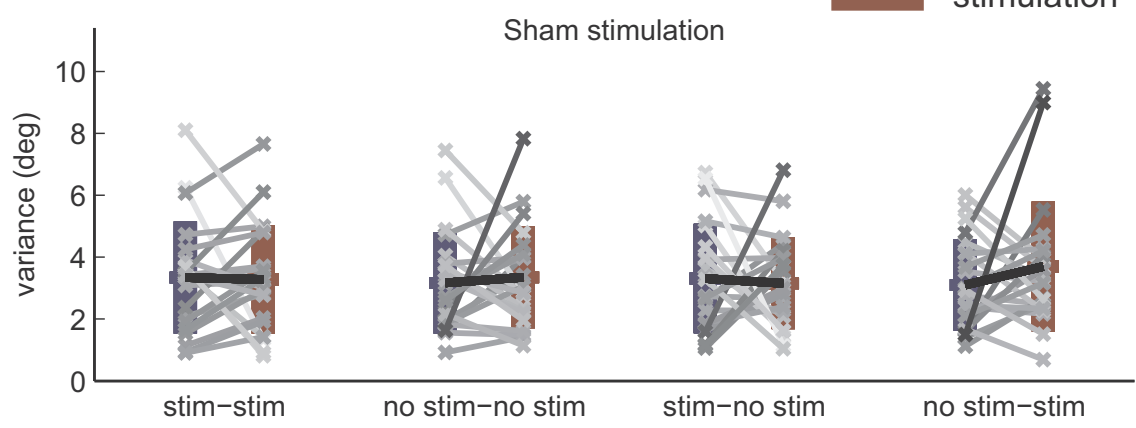

Fig. 3. Group averages for the variance with actual stimulation (A) or sham (control) stimulation (B). Vertical colored bars denote \pm 1 standard deviation from the mean. Gray lines are individual subject data; the black line denotes the mean. The reduction in variance, and thus increase in precision in the no stimstim condition, was significantly different between the actual and sham stimulation conditions $(\mathrm{p}=0.017)$. 

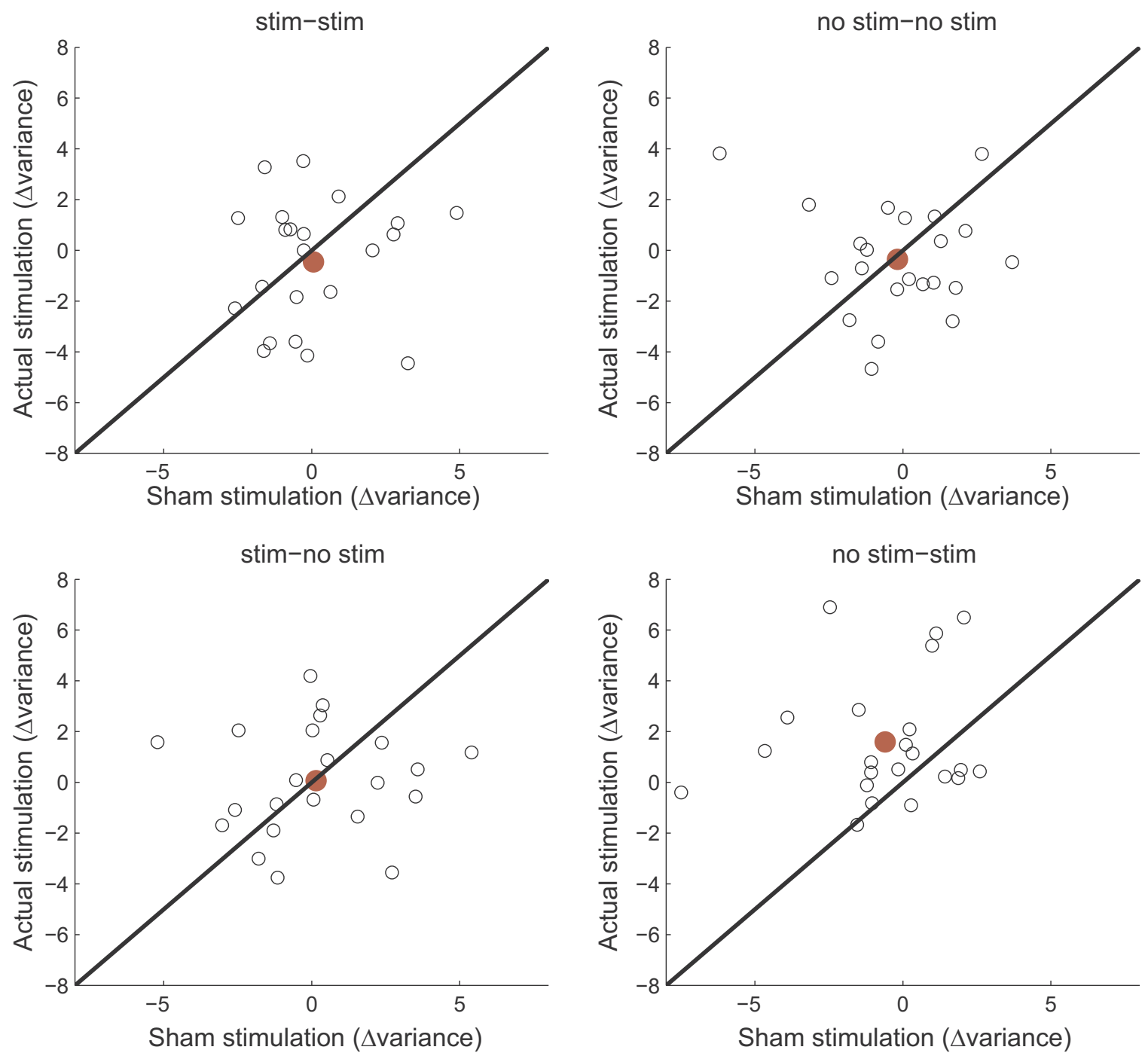

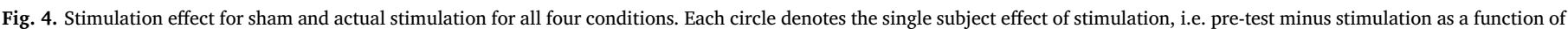

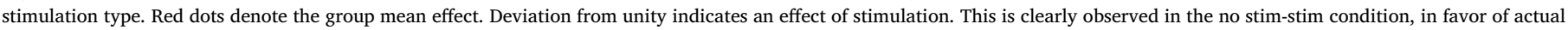
stimulation. (For interpretation of the references to color in this figure legend, the reader is referred to the web version of this article.)

\section{Discussion}

The aim of the present study was to investigate the role of alpha oscillations in the remapping process for whole-body spatial updating. While subjects were fixating straight ahead, we presented targets at locations that had to be remembered across whole body motion, either within one hemifield (do not cross the gaze/body midline) or across hemifields (where the remembered location crossed relative to the midline). By applying $10 \mathrm{~Hz}$ tACS at an intensity of $1 \mathrm{~mA}$ over either the left- or right parieto-occipital area, we compared updating performance to a baseline measurement just before stimulation, and a sham condition in a separate session.

We found no stimulation-related effects for the updating gain, irrespective of whether the remapping was across or within hemispheres. This suggests that alpha band oscillations do not directly affect the transfer of target representations during remapping. However, updating precision was enhanced when a target representation had to be internally remapped to the stimulated hemisphere, but not in other remapping conditions. This finding points to a role of alpha band oscillation in protecting the updated representation from distortions.

Based on previous alpha-band tACS using a parieto-occipital montage (Helfrich et al., 2014; Neuling et al., 2013; Zaehle et al., 2010), we assume that our current parieto-occipital to vertex montage increased alpha-band power in the underlying areas during tACS, even though we did not record EEG during tACS. Furthermore, increases in alpha have generally been associated with the selective inhibition of neuronal populations in the visual areas (Klimesch et al., 2007; Sauseng et al., 2005; Thut et al., 2006). Within this framework, we interpret the increase in precision in the no stim-stim condition as follows: First, there was an increase in parieto-occipital areas ipsilateral to the initial target, which is beneficial to disengage this hemisphere from interfering with the initial target processing in the opposite hemisphere. In our previous study we have shown that alpha-band power is reduced in this hemisphere after presentation of the initial target (Gutteling et al., 2015), which would thus be facilitated by inhibiting the other hemisphere. In other words, there is a positive effect on visual encoding due to reduced sensitivity of the non-relevant hemisphere (Rihs et al., 2007). This interpretation concurs with similar observations in paradigms with distractor stimuli (Kelly et al., 2006; Worden et al., 2000).

In our previous study we also found evidence for alpha-power increases in the hemisphere contralateral to the updating target location. Even though this may seem counterintuitive for the hemisphere that is engaged, the alpha oscillations may actually help to preserve the target representation by blocking further inputs affecting its representation (Gutteling and Medendorp, 2016) and thereby reducing interference effects for the remembered target. In support, alpha-band increases 
have also been observed in the context of working memory retention (Jensen et al., 2002; Rihs et al., 2009). If the increase in alpha activity is further enhanced by tACS it could explain why the quality of the updated representation (i.e., precision) is increased. Importantly, no precision effect was observed for targets appearing in the stimulated hemifield, where the remapped target was in the non-stimulated hemifield. This argues for a role of alpha band oscillations in reducing interference for the spatial target representation that is being remapped. However, because there were no $10 \mathrm{~Hz}$ tACS effects on the updating gain, we could not demonstrate that alpha-band oscillations are directly involved in the remapping process itself.

The tACS primarily affected the remapped (remembered) target, but not the visually presented initial target. This is consistent with neuropsychological findings from optic ataxia patients with parieto-occipital lesions (Blangero et al., 2011), showing that these patients are impaired when reaching for a target that has been remapped to the contralesional field, but not when the initial target appears in the contralesional field.

Of note, in the current study, the increase in precision did not occur when targets had to be shifted within the stimulated hemisphere. It is possible that the improved precision due to tACS relies on two effects: (1) ipsilateral alpha increase during encoding and (2) contralateral increase during interhemispheric remapping. It may be that both these effects have to be present to produce a noticeable benefit during spatial updating. The within-hemisphere conditions (stim-stim and no stim-no stim), as well as the stim-no stim condition lack contribution of both these effects and thus, no observable effect is present.

Upon visual inspection of the data, it became apparent that the baseline measurement, i.e. the pre-test performance before the real or sham tACS session, was not equal across conditions. As the subjects, and the experimenter were blind to the ensuing stimulation type (real of sham tACS), no differences in baseline performance were expected. Precautions were taken to minimize week-to-week variation by testing at the same time of day in both weeks. As mentioned in the Results section, these state-dependent effects could not be explained by session order effects (counterbalancing), or gender balancing. Because some variation from week to week is common, we measured this variation in order to compensate for it, by examining the difference in performance within sessions, and comparing these differences across stimulation conditions.

Our results also show that $10 \mathrm{~Hz}$ tACS does not affect the gain of the spatial update. Previous studies from our lab (Gutteling et al., 2015; Gutteling and Medendorp, 2016) have shown that alpha-band activity in central parietal areas correlates with spatial updating behavior, i.e. updating gain. Given that tACS was applied over more lateralized occipital areas, this may not have affected central parietal processing. Alternatively, it may be that alpha-band activity is not directly involved in the remapping process, but is observed after the remapping process, enabling a stable spatial representation.

Finally, our current findings with $10 \mathrm{~Hz}$ tACS do not imply that effects in this paradigm are restricted to this frequency. Given that we only tested using $10 \mathrm{~Hz}$, we cannot make claims on the frequency specificity of the observed effects. However, with this $10 \mathrm{~Hz}$ stimulation, our observations suggest that the oscillations may not affect the transfer of target representations during remapping, but do reflect the quality of the representations that are encoded, stored, updated and maintained.

\section{Acknowledgements}

This work was supported by EU-FP7-FET SpaceCog Grant 600785, the European Research Council (EU-ERC-283567), and the Netherlands Organization for Scientific Research (NWO-VICI: 453-11-001).

\section{References}

Blangero, A., Khan, A., Rode, G., Rossetti, Y., Pisella, L., 2011. Dissociation between intentional and automatic remapping: different levels of inter-hemispheric transfer. Vision. Res. 51, 932-939. http://dx.doi.org/10.1016/j.visres.2011.01.012.

Brinkman, L., Stolk, A., Marshall, T.R., Esterer, S., Sharp, P., Dijkerman, H.C., de Lange, F.P., Toni, I., 2016. Independent causal contributions of alpha- and beta-band oscillations during movement selection. J. Neurosci. 36, 8726-8733. http://dx.doi.org/ 10.1523/JNEUROSCI.0868-16.2016.

Cecere, R., Rees, G., Romei, V., 2015. Individual differences in alpha frequency drive crossmodal illusory perception. Curr. Biol. 25, 231-235. http://dx.doi.org/10.1016/ j.cub.2014.11.034.

Clemens, I.A.H., Selen, L.P.J., Koppen, M., Medendorp, W.P., 2012. Visual stability across combined eye and body motion. J. Vis. 12. http://dx.doi.org/10.1167/12.12.8.

Colby, C.L., Duhamel, J.R., Goldberg, M.E., 1995. Oculocentric spatial representation in parietal cortex. Cereb. Cortex 5, 470-481. http://dx.doi.org/10.1093/cercor/5.5. 470. (New York, NY 1991).

Fründ, I., Haenel, N.V., Wichmann, F.A., 2011. Inference for psychometric functions in the presence of nonstationary behavior. J. Vis. 11. http://dx.doi.org/10.1167/11. 6.16 .

Gutteling, T.P., Medendorp, W.P., 2016. Role of alpha-band oscillations in spatial updating across whole body motion. Front. Psychol. 7. http://dx.doi.org/10.3389/ fpsyg.2016.00671.

Gutteling, T.P., Selen, L.P.J., Medendorp, W.P., 2015. Parallax-sensitive remapping of visual space in occipito-parietal alpha-band activity during whole-body motion. J. Neurophysiol. 113, 1574-1584. http://dx.doi.org/10.1152/jn.00477.2014.

Helfrich, R.F., Schneider, T.R., Rach, S., Trautmann-Lengsfeld, S.A., Engel, A.K., Herrmann, C.S., 2014. Entrainment of brain oscillations by transcranial alternating current stimulation. Curr. Biol. 24, 333-339. http://dx.doi.org/10.1016/j.cub.2013. 12.041.

Herrmann, C.S., Rach, S., Neuling, T., Strüber, D., 2013. Transcranial alternating current stimulation: a review of the underlying mechanisms and modulation of cognitive processes. Front. Hum. Neurosci. 7, 279. http://dx.doi.org/10.3389/fnhum.2013. 00279.

Jensen, O., Gelfand, J., Kounios, J., Lisman, J.E., 2002. Oscillations in the alpha band $(9-12 \mathrm{~Hz})$ increase with memory load during retention in a short-term memory task. Cereb. Cortex 12, 877-882.

Jensen, O., Mazaheri, A., 2010. Shaping functional architecture by oscillatory alpha activity: gating by inhibition. Front. Hum. Neurosci. 4, 186. http://dx.doi.org/10. 3389/fnhum.2010.00186.

Kar, K., Krekelberg, B., 2014. Transcranial alternating current stimulation attenuates visual motion adaptation. J. Neurosci. 34, 7334-7340. http://dx.doi.org/10.1523/ JNEUROSCI.5248-13.2014.

Kelly, S.P., Lalor, E.C., Reilly, R.B., Foxe, J.J., 2006. Increases in alpha oscillatory power reflect an active retinotopic mechanism for distracter suppression during sustained visuospatial attention. J. Neurophysiol. 95, 3844-3851. http://dx.doi.org/10.1152/ jn.01234.2005.

Klier, E.M., Angelaki, D.E., 2008. Spatial updating and the maintenance of visual constancy. Neuroscience 156, 801-818. http://dx.doi.org/10.1016/j.neuroscience.2008. 07.079.

Klimesch, W., Sauseng, P., Hanslmayr, S., 2007. EEG alpha oscillations: the inhibitiontiming hypothesis. Brain Res. Rev. 53, 63-88. http://dx.doi.org/10.1016/j. brainresrev.2006.06.003.

Medendorp, W.P., 2011. Spatial constancy mechanisms in motor control. Philos. Trans. R. Soc. B Biol. Sci. 366, 476-491. http://dx.doi.org/10.1098/rstb.2010.0089.

Medendorp, W.P., Goltz, H.C., Vilis, T., Crawford, J.D., 2003. Gaze-centered updating of visual space in human parietal cortex. J. Neurosci. 23, 6209-6214.

Merriam, E.P., Genovese, C.R., Colby, C.L., 2003. Spatial updating in human parietal cortex. Neuron 39, 361-373.

Morris, A.P., Chambers, C.D., Mattingley, J.B., 2007. Parietal stimulation destabilizes spatial updating across saccadic eye movements. Proc. Natl. Acad. Sci. USA 104, 9069-9074. http://dx.doi.org/10.1073/pnas.0610508104.

Neuling, T., Rach, S., Herrmann, C.S., 2013. Orchestrating neuronal networks: sustained after-effects of transcranial alternating current stimulation depend upon brain states. Front. Hum. Neurosci. 7, 161. http://dx.doi.org/10.3389/fnhum.2013.00161.

Rihs, T.A., Michel, C.M., Thut, G., 2009. A bias for posterior alpha-band power suppression versus enhancement during shifting versus maintenance of spatial attention. Neuroimage 44, 190-199. http://dx.doi.org/10.1016/j.neuroimage.2008.08.022.

Rihs, T.A., Michel, C.M., Thut, G., 2007. Mechanisms of selective inhibition in visual spatial attention are indexed by alpha-band EEG synchronization. Eur. J. Neurosci. 25, 603-610. http://dx.doi.org/10.1111/j.1460-9568.2007.05278.x.

Ruhnau, P., Neuling, T., Fuscá, M., Herrmann, C.S., Demarchi, G., Weisz, N., 2016. Eyes wide shut: transcranial alternating current stimulation drives alpha rhythm in a state dependent manner. Sci. Rep. 6, 27138. http://dx.doi.org/10.1038/srep27138.

Sauseng, P., Klimesch, W., Stadler, W., Schabus, M., Doppelmayr, M., Hanslmayr, S., Gruber, W.R., Birbaumer, N., 2005. A shift of visual spatial attention is selectively associated with human EEG alpha activity. Eur. J. Neurosci. 22, 2917-2926. http:// dx.doi.org/10.1111/j.1460-9568.2005.04482.x.

Schutter, D.J.L.G., 2015. Cutaneous retinal activation and neural entrainment in transcranial alternating current stimulation: a systematic review. Neuroimage. http://dx. doi.org/10.1016/j.neuroimage.2015.09.067.

Schutter, D.J.L.G., Wischnewski, M., 2016. A meta-analytic study of exogenous oscillatory electric potentials in neuroenhancement. Neuropsychologia. http://dx.doi.org/10. 1016/j.neuropsychologia.2016.04.011.

Sommer, M.A., Wurtz, R.H., 2008. Visual perception and corollary discharge. Perception 
37, 408-418.

Thut, G., Nietzel, A., Brandt, S.A., Pascual-Leone, A., 2006. Alpha-band electroencephalographic activity over occipital cortex indexes visuospatial attention bias and predicts visual target detection. J. Neurosci. 26, 9494-9502. http://dx.doi.org/ 10.1523/JNEUROSCI.0875-06.2006.

Van Der Werf, J., Buchholz, V.N., Jensen, O., Medendorp, W.P., 2013. Reorganization of oscillatory activity in human parietal cortex during spatial updating. Cereb. Cortex 23, 508-519. http://dx.doi.org/10.1093/cercor/bhr387.

Vossen, A., Gross, J., Thut, G., 2015. Alpha power increase after transcranial alternating current stimulation at alpha frequency (a-tACS) reflects plastic changes rather than entrainment. Brain Stimul. 8, 499-508. http://dx.doi.org/10.1016/j.brs.2014.12. 004.

Worden, M.S., Foxe, J.J., Wang, N., Simpson, G.V., 2000. Anticipatory biasing of visuospatial attention indexed by retinotopically specific alpha-band electroencephalography increases over occipital cortex. J. Neurosci. 20, RC63.

Zaehle, T., Rach, S., Herrmann, C.S., 2010. Transcranial alternating current stimulation enhances individual alpha activity in human EEG. PLoS One 5, e13766. http://dx.doi. org/10.1371/journal.pone.0013766. 